\title{
Danshensu inhibits the IL-1 $\beta$-induced inflammatory response in chondrocytes and osteoarthritis possibly via suppressing NF-KB signaling pathway
}

\author{
Zhixian Xu ${ }^{1,2,3}$, Tie Ke $e^{1,2}$, Yongfa Zhang ${ }^{1,2}$, Licheng Guo ${ }^{1,2}$, Feng Chen ${ }^{1,2,3^{*}}$ (D) and Wubing He $\mathrm{e}^{1,2,3^{*}}$
}

\begin{abstract}
Purpose: Osteoarthritis $(\mathrm{OA})$ is the most common inflammatory disease associated with pain and cartilage destruction. Interleukin (IL)-1 $\beta$ is widely used to induce inflammatory response in OA models. This study aimed to explore the role of Danshensu (DSS) in IL-1 $\beta$-induced inflammatory responses in OA.

Methods: IL-1 $\beta$ was used to induce chondrocyte inflammation. Cell viability was evaluated by Cell Counting Kit-8 (CCK-8) assay. IL-6, COX-2, TNF-a, and iNOS mRNA levels were detected by qRT-PCR. MMP3, MMP13, ADAMTS4, ADAMTS5, Aggrecan, Collagen, p-IKBa, and p-p65 protein levels were detected by Western blot. An OA mouse model was established by surgical destabilization of the medial meniscus (DMM), and the Osteoarthritis Research Society International (OARSI) score was evaluated by H\&E staining.

Results: DSS did not affect the levels of inflammatory indicators including IL-6, COX-2, TNF-a, iNOS, PEG2, and NO but suppressed COX-2 and iNOS protein expression in IL-1 $\beta$ treated chondrocytes. In addition, DSS downregulated IL-1 $\beta$ enhanced expression of MMP3, MMP13, ADAMTS4, and ADAMTS5 and upregulated aggrecan and collagen expression. Moreover, DSS significantly inhibited IL-1 $\beta$-induced phosphorylation of $\mathrm{p}-\mathrm{k} \mathrm{kB}$ a and p-p65 in a dose-dependent manner in chondrocytes, suggesting it plays a role in the NF-kB signaling pathway. Furthermore, DSS significantly reduced DMM-induced cartilage OARSI score in mice, further demonstrating its protective role in OA progression in vivo.
\end{abstract}

Conclusions: Our study revealed the protective role of DSS in OA, suggesting that DSS might act as a potential treatment for OA.

Keywords: Osteoarthritis, Danshensu, IL-1 $\beta$, Inflammatory response, NF-kB

\section{Introduction}

Osteoarthritis (OA) has become a common joint disease, which is characterized by pain and could lead to disability in the adult population, affecting approximately 240 million people worldwide (Rim et al. 2020; Nelson 2018).

\footnotetext{
*Correspondence: FengChenmedicine@163.com; uj1889@163.com

1 Shengli Clinical Medical College of Fujian Medical University, Fuzhou 350004, Fujian Province, People's Republic of China

Full list of author information is available at the end of the article
}

Increasing evidence indicates that approximately $30 \%$ of OA patients may undergo neuropathic pain (Schomberg et al. 2012). OA is a disease with cartilage degeneration, chondrocyte inflammation, and subchondral sclerosis of the whole joint, of which cartilage degeneration and inflammation response play essential roles in OA progression (Goldring and Goldring 2016). Low-level inflammation can contribute to the degenerative changes and development of peripheral sensitization and nociceptive

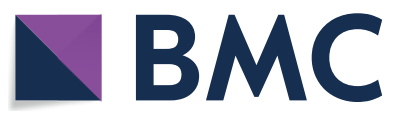

(c) The Author(s) 2021. Open Access This article is licensed under a Creative Commons Attribution 4.0 International License, which permits use, sharing, adaptation, distribution and reproduction in any medium or format, as long as you give appropriate credit to the original author(s) and the source, provide a link to the Creative Commons licence, and indicate if changes were made. The images or other third party material in this article are included in the article's Creative Commons licence, unless indicated otherwise in a credit line to the material. If material is not included in the article's Creative Commons licence and your intended use is not permitted by statutory regulation or exceeds the permitted use, you will need to obtain permission directly from the copyright holder. To view a copy of this licence, visit http://creativecommons.org/licenses/by/4.0/. 
pain (Krustev et al. 2015; McDougall et al. 2009). Many therapeutic strategies for OA mainly aimed to alleviate inflammatory pain, reduce stiffness, maintain cartilage functional capacities, and improve patients' quality of life (Felson 2006; Conaghan et al. 2019). However, identifying effective treatment strategies in this common chronic OA, specifically OA pain, is still a challenge.

Aggrecan and collagen are the main components of the extracellular matrix (ECM). Downregulation of their expression leads to cartilage degradation (Pattoli et al. 2005). Disintegrin and metalloproteinase with thrombospondin motifs (ADAMTS) enzymes, especially ADAMTS- 4 and ADAMTS-5, are considered the primary aggrecanases with the ability to cleave aggrecans (Stanton 2005). In addition, IL-1 $\beta$ can induce cartilage degradation by promoting the expression of matrix metalloproteinases (MMPs) in chondrocytes (Yin and Lei 2018; Zhang 2019). Previous studies have shown that inflammatory factors like IL- $1 \beta$ play a crucial role in OA progression by regulating nuclear factor-kappa B (NF$\kappa B)$ (Jotanovic et al. 2012; Berenbaum 2004). NF- $\kappa B$ is a dimer composed of two proteins, $\mathrm{p} 65$ and $\mathrm{I} \kappa \mathrm{B} \alpha$, at the resting state. IкB $\alpha$ phosphorylation and subsequent degradation lead to p65 phosphorylation, activation, and translocation from the cytoplasm to the nucleus, where it promotes the transcription of its target genes (Mendes et al. 2002), including many inflammation-related genes such as MMPs, iNOS, and IL-6 (Mitchell and Carmody 2018). NF- $\mathrm{kB}$ signaling pathway has been well identified to participate in the inflammatory progress and considered a potential target in OA treatment (Saklatvala 2007).

In the last decades, replacement surgery has been the primary approach for OA treatment to control the symptoms (Brosseau 2003). Based on the identification that inflammation is responsible for OA progression, specifically OA pain, anti-inflammation substances are potentially applied for treating OA to inhibit chondrocyte inflammation. For instance, trans-cinnamaldehyde (TCA) inhibits IL-1 $\beta$-stimulated inflammation in chondrocytes by suppressing NF- $\mathrm{KB}$ and $\mathrm{p} 38$-JNK pathways and exhibits chondrocyte protective effects in a rat $\mathrm{OA}$ model (Xia 2019). Schisandrin B ameliorates chondrocyte inflammation and OA progression through inhibiting NF- $\mathrm{KB}$ activation and MAPK signaling pathway (Ran 2018). In addition, nonsteroidal anti-inflammatory drugs (NSAIDs) are widely applied for OA treatment to reduce chronic pain (Argoff 2011). Despite these advantages, it is necessary to identify safer and more effective therapeutic agents for OA.

Danshensu (DSS) is a bioactive agent isolated from an edible traditional Chinese medicine salvia miltiorrhiza (dan shen) (Cao 2019). DSS has been reported to have an anti-inflammatory effect involving TLR2 (Toll-like receptor 2) and macrophages through the NF- $\mathrm{kB}$ signaling pathway (Ye 2020). In addition, DSS can also significantly ameliorate colon inflammation in dextran sulfate sodium-induced colitis (Wen 2013). In chronic kidney disease, the combination of rhein $(\mathrm{RH})$, a natural chondroprotective agent, and DSS shows a better protective effect through improving renal function, blood supply, fibrotic degree, and suppressing pro-inflammatory cytokines via deactivating NK- $\mathrm{kB}$ signaling pathway (Guan 2015). However, the roles of DSS in OA and its underlying specific mechanism remain unclear.

Here, we demonstrated that DSS downregulated IL-1 $\beta$ induced over-production of inflammatory cytokines including nitric oxide (NO), tumor necrosis factor (TNF)- $\alpha$, prostaglandin $\mathrm{E}_{2}$ (PGE2), and IL-6, as well as the expression of cyclooxygenase-2 (COX-2), inducible NO synthase (iNOS), MMP3, MMP13, ADAMTS4, and ADAMTS5 in chondrocytes. Meanwhile, DSS promoted IL-1 $\beta$-induced defective expression of aggrecan and collagen in chondrocytes. Specifically, our results indicated that DSS inhibited IL- $1 \beta$-activated NF- $\mathrm{kB}$ signaling pathway in chondrocytes. Overall, our study indicated that DSS could efficiently prevent OA progression in vivo and provided a potential treatment strategy for $\mathrm{OA}$ treatment.

\section{Materials and methods}

\section{Animal experiments}

A total of sixty 10-12-week-old C57BL/6 male mice from the Animal Center of Chinese Academy of Sciences Shanghai, China, were used in the study. The OA mouse model was established using surgical destabilization of the medial meniscus (DMM), as previously described (Liu 2020). These mice were randomly divided into three groups, namely the sham group, DMM group, and DMM + DSS group, with 10 mice per group. The mice in the DSS group were then intraperitoneally injected with $40 \mathrm{mg} / \mathrm{kg}$ DSS, and mice in the Sham and DMM group were injected with PBS as the control. The mice were sacrificed by injecting overdose pentobarbital sodium at the end of the 8th week. The animal experiment was performed according to the National Institutes of Health Guide for Care and Use of Laboratory Animals and approved by Fujian Provincial Hospital.

\section{Chondrocyte isolation and culture}

Chondrocytes were isolated from C57BL/6 male mice as previously described (Xu 2020). The isolated chondrocytes were cultured in low-glucose DMEM containing $10 \% \mathrm{FBS}, 100 \mathrm{U} / \mathrm{mL}$ penicillin, and $100 \mathrm{mg} / \mathrm{mL}$ streptomycin. Danshensu (purity 99\%) was purchased from the National Institute for the Control of Pharmaceutical and Biological Products (Beijing, China) and dissolved 
in DMSO to obtain the desired concentrations. When needed, cells were treated with $10 \mathrm{ng} / \mathrm{ml} \mathrm{IL-1} \beta$ and different concentrations of DSS for different times as indicated and collected for subsequent experiments.

\section{Cell viability}

Chondrocytes were plated into 96-well plates at a density of $\sim 5000$ cells/well. Cells were treated with different concentrations of DSS $(0,1.25,2.5,5,10,20$, and $40 \mu \mathrm{M})$ for $24 \mathrm{~h}$ or $48 \mathrm{~h}$, respectively, collected, and subjected to cell viability assay using Cell Counting Kit-8 (CCK-8) solution (Dojindo Molecular Technologies, Inc.). The absorbance at $450 \mathrm{~nm}$ was detected as an indicator of the cell viability using a microplate reader (Bio-Rad, Hercules, CA, USA).

\section{qRT-PCR}

Total RNA of chondrocytes was extracted with TRIzol reagent according to the manufacturer's instructions. Approximately $1 \mu \mathrm{g}$ RNA was transcribed to cDNA using Double-Strand cDNA Synthesis Kits, and qRT-PCR was performed using the SYBR-based CFX96 Real-Time PCR System. The relative expression change of targets was analyzed using the $2^{-\Delta \Delta \mathrm{Ct}}$ method (Schmittgen and Livak 2008), with $\beta$-actin as the internal reference. The primers used in this study were TNF- $\alpha$ forward $5^{\prime}$-CAG GCGGTGCCTATGTCTC- $3^{\prime}$ and reverse $5^{\prime}$-CGATCA CCCCGAAGTTCAGTAG- $3^{\prime}$, IL-6 forward 5'-TACCAC TTCA CAAGTCGGAGGC-3' and reverse $5^{\prime}$-CTGCAA GTGCATCATCGTTGTTC-3, iNOS forward $5^{\prime}$-CTC TTCGACGACCCAGAAAAC-3' and reverse 5'-CAA GGCCATGAA GTGAGGCTT- 3', Cox-2 forward5'CACCCTGACATAGACAGTGAAAG- $3^{\prime}$ and reverse $5^{\prime}$-CTGGGTCACGTTGGATGAGG- ${ }^{\prime}$, as well as $\beta$-actin forward $5^{\prime}$-AGCCA TGTACGTAGCCAT CC-3' and reverse $5^{\prime}$-CTCTCAGCAGTGGTGGTGAA- $3^{\prime}$.

\section{Western blot}

The total proteins from chondrocytes were extracted using RIPA lysis buffer, and protein concentration was determined with the $\mathrm{BCA}$ protein assay kit according to the manufacturer's instruction. Approximately $40 \mu \mathrm{g}$ protein samples were separated by $12 \%$ SDS-PAGE and transferred onto PVDF membranes. After blocking with $5 \%$ nonfat milk, the membranes were induced with primary antibodies against COX-2 (1:1000, Abcam), iNOS (1:1000, Abcam), MMP-3 (1:1000, Abcam), MMP13 (1:1000, Abcam), ADAMTS-4 (1:1000, Abcam), ADAMTS-5 (1:1000, Abcam), aggrecan (1:1000, Abcam), collagen II (1:1000, Abcam), p65 (1:2000, Abcam), p-p65 (1:2000, Abcam), IkB $\alpha$ (1:2000, Abcam), p-IkB $\alpha$ (1:2000, Abcam), lamin B (1:1000, Abcam) and $\beta$-actin (1:5000, Abcam) overnight at $4{ }^{\circ} \mathrm{C}$. Then the membranes were washed and incubated with HRP-conjugated secondary antibodies (1:3000) for $2 \mathrm{~h}$. Finally, signals on the membranes were detected using the Enhanced Chemiluminescence (ECL) kit and quantified by the Quantity ONE software (Bio-Rad, USA).

\section{Histological analysis}

Knee joints were isolated from mice and fixed with $4 \%$ formaldehyde. Calcium in the knee joints was removed using a decalcifying solution for 21 days. At last, the knee joints were embedded in paraffin and coronally sectioned. The sections were stained with Safranin O or hematoxylin \& eosin (H\&E) as previously described (Jia 2020) and scored using the Osteoarthritis Research Society International (OARSI) scoring system to determine the extent of cartilage deterioration as previously reported (Pritzker 2006). Grade 0 was for intact surface and cartilage; Grade 1 for intact surface only; Grade 2 for surface discontinuity, Grade 3 for vertical fissures; Grade 4 for erosion, Grade 5 for denudation, and Grade 6 for deformation.

\section{Immunofluorescence assay}

Chondrocytes that had been pretreated with $10 \mu \mathrm{M}$ DSS for $1 \mathrm{~h}$ and then treated with $10 \mathrm{ng} / \mathrm{mL} \mathrm{IL}-1 \beta$ for $30 \mathrm{~min}$ were plated on glass coverslips. After fixation in cold methanol, cells were incubated with primary antibody against collagen II (1:500) overnight at $4{ }^{\circ} \mathrm{C}$, and then with fluorescein isothiocyanate-conjugated secondary antibody for $1 \mathrm{~h}$ and DAPI solution for $5 \mathrm{~min}$. After that, cells were observed and photographed using a Leica fluorescence microscope.

\section{Data analysis}

All experiments were performed at least three independent times. Data were presented as mean \pm standard deviation (SD). The difference between two groups was analyzed by Student's t-tests and among multiple groups were analyzed with one-way ANOVA, with $\mathrm{p}<0.05$ as the significant threshold.

\section{Results \\ Low-dose DSS showed no cytotoxicity to osteoarthritic chondrocytes}

The chemical structure of DSS was shown in Fig. 1A. To explore its role in OA progression, we first isolated chondrocytes from C57BL/6 male mice. The morphology of the isolated primary chondrocytes was evaluated and shown in Fig. 1B, which indicated that chondrocytes were successfully isolated and could be applied for subsequent experiments. We then treated the chondrocytes with DSS at different concentrations $(0,1.25,2.5,5,10$, 20 , and $40 \mu \mathrm{M}$ ) for 24 and $48 \mathrm{~h}$, respectively. The CCK- 8 
(A)<smiles>O=C(O)[C@H](O)Cc1ccc(O)c(O)c1</smiles>

(C)

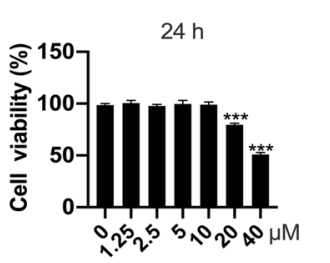

(B)

(D)

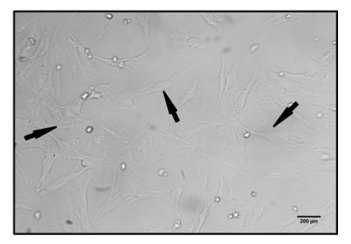

$48 \mathrm{~h}$

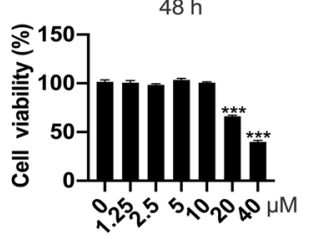

Fig. 1 The cytotoxicity of DSS on chondrocytes. A The structure of DSS. B The morphology of primary chondrocytes. Scale bar $=200 \mu \mathrm{m}$. C, D Chondrocyte viability was detected by the CCK-8 kit after treatment with different concentrations of DSS for $24 \mathrm{~h}(\mathbf{C})$ and $48 \mathrm{~h}$ (D), respectively. ${ }^{* * *} \mathrm{p}<0.001$

assay indicated that chondrocyte viability was significantly reduced after treatment with 20 and $40 \mu \mathrm{M}$ DSS for $24 \mathrm{~h}(\mathrm{p}<0.001)$ (Fig. 1C) and $48 \mathrm{~h}(\mathrm{p}<0.001)$ (Fig. 1D), respectively, but not after treatment with $<10 \mu \mathrm{M}$ DSS, indicating that DSS at low concentration $(\leq 10 \mu \mathrm{M})$ exhibited no obvious cytotoxicity to chondrocytes. Hence, we selected DSS at 2.5, 5, and $10 \mu \mathrm{M}$ to explore its role in OA progression.

\section{DSS inhibited IL-1 $\beta$-induced chondrocyte inflammation}

To investigate the effects of DSS in IL- $1 \beta$-induced chondrocyte inflammation, chondrocytes were pretreated with DSS for $24 \mathrm{~h}$ at $2.5,5$, and $10 \mu \mathrm{M}$, respectively, and treated with $10 \mathrm{ng} / \mathrm{ml} \mathrm{IL-1} \beta$ for $2 \mathrm{~h}$. qRT-PCR analyses showed that DSS treatment attenuated IL- $1 \beta$-induced expression of IL- 6 , COX-2, TNF- $\alpha$, and iNOS in a dosedependent manner (all $\mathrm{p}<0.001$ ) (Fig. 2A). Similarly, Western blot results also confirmed the dose-dependent downregulatory effects of DSS on IL-1 $\beta$-elevated protein levels of inflammation-related indicators, including COX-2 and iNOS ( $\mathrm{p}<0.01$ ) (Fig. 2B). Further, the production of NO, PGE2, IL-6, and TNF- $\alpha$ in the culture supernatant was detected by ELISA kit, and the results showed that DSS treatment significantly inhibited IL- $1 \beta$-induced secretion of NO, PGE2, IL-6, and TNF- $\alpha$ (all $p<0.001$ ) (Fig. 2C). These results indicated that DSS inhibited IL-1 $\beta$-induced chondrocyte inflammation.

\section{DSS protected chondrocytes against IL-1 $\beta$-caused ECM degradation}

To explore the role of DSS pretreatment on ECM degradation, we detected the protein level of ECM-related

genes in IL-1 $\beta$-treated chondrocytes by Western blot. We found that IL-1 $\beta$ treatment decreased aggrecan and collagen II expressions while significantly promoted ADAMTS-4, ADAMTS-5, MMP-3, and MMP-13 expressions. However, these IL- $1 \beta$-induced changes were significantly reversed by DSS pretreatment, meaning that DSS pretreatment increased aggrecan and collagen II expression and reduced ADAMTS-4, ADAMTS-5, MMP-3, and MMP-13 expression $(\mathrm{p}<0.05)$ (Fig. 3A, B). In addition, immunofluorescence assay indicated that DSS significantly rescued IL- $1 \beta$-stimulated collagen II degradation $(\mathrm{p}<0.001)$ and inhibited IL- $1 \beta$-induced MMP3 overexpression in chondrocytes $(\mathrm{p}<0.05)$ (Fig. 3C, D). These results indicated that DSS efficiently protected chondrocytes against IL-1 $\beta$-induced ECM degradation.

\section{DSS inhibited IL-1 $\beta$-induced activation of NF-KB pathway in chondrocytes}

We next evaluated the effects of DSS on the NF-kB pathway. The results indicated that IL- $1 \beta$ significantly increased p-p65 and p-IкB $\alpha$ levels in chondrocytes, leading to I $\mathrm{KB} \alpha$ degradation. Meanwhile, DSS dramatically inhibited IL-1 $\beta$-elevated p-IкB $\alpha$ and p-p65 levels in a dose-dependent manner $(\mathrm{p}<0.05)$ (Fig. 4A, B).

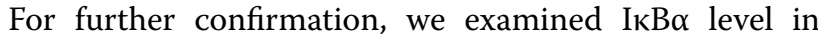
the cytoplasm and p65 in the nucleus of IL-1 $\beta$-treated chondrocytes by Western blot. The results indicated that IL- $1 \beta$ treatment reduced I $\kappa \beta \alpha$ level in the cytoplasm and promoted p65 translocating to the nucleus. These effects were significantly reversed by DSS pretreatment in a dose-dependent manner $(\mathrm{p}<0.01)$ (Fig. $4 \mathrm{C}, \mathrm{D})$, indicating that DSS pretreatment efficiently inhibited IL-1 $\beta$-induced activation of the NF- $\mathrm{kB}$ pathway in OA chondrocytes.

\section{DSS attenuated OA progression in the OA mouse model}

To confirm whether DSS played a protective role in OA progression in vivo, the surgical mouse OA model was established, and DSS was applied. The results showed that mice in the sham group had smooth and intact cartilage surface while mice in the DMM group had destructed cartilage surface, eroded cartilage, and apparent hypocellularity. Moreover, DSS suppressed cartilage degradation observed in the DMM group (Fig. 5A). Consistent with the observed phenotypes in the cartilage of mice, DSS significantly reduced cartilage OARSI score elevated by DMM ( $\mathrm{p}<0.001)$ (Fig. 5B). These data demonstrated that DSS efficiently attenuated OA progression in vivo.

\section{Discussion}

Danshensu, a herbal preparation used in traditional Chinese medicine, has been reported to exhibit protective effects in various human diseases, including Parkinson's 

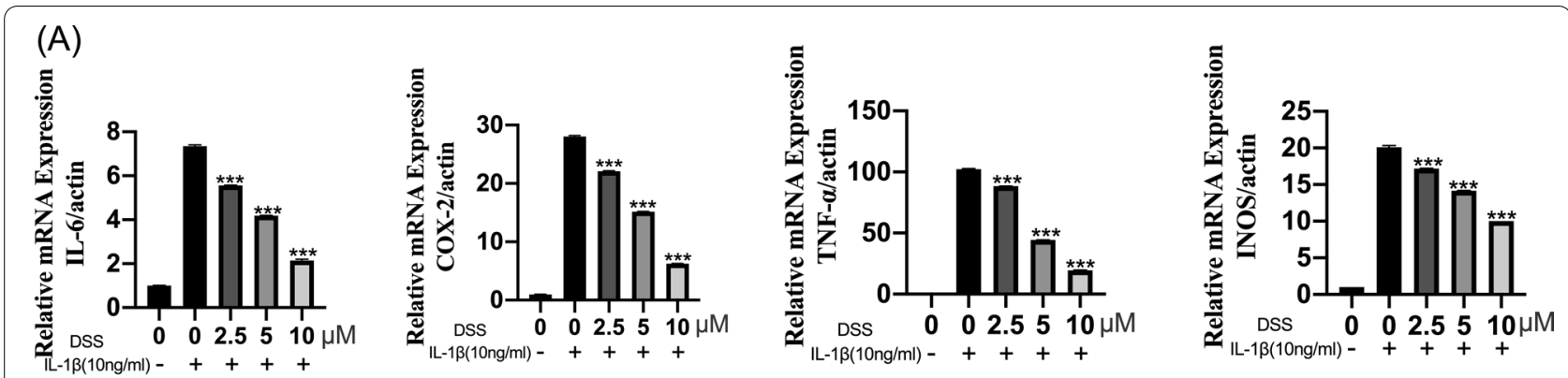

(B)
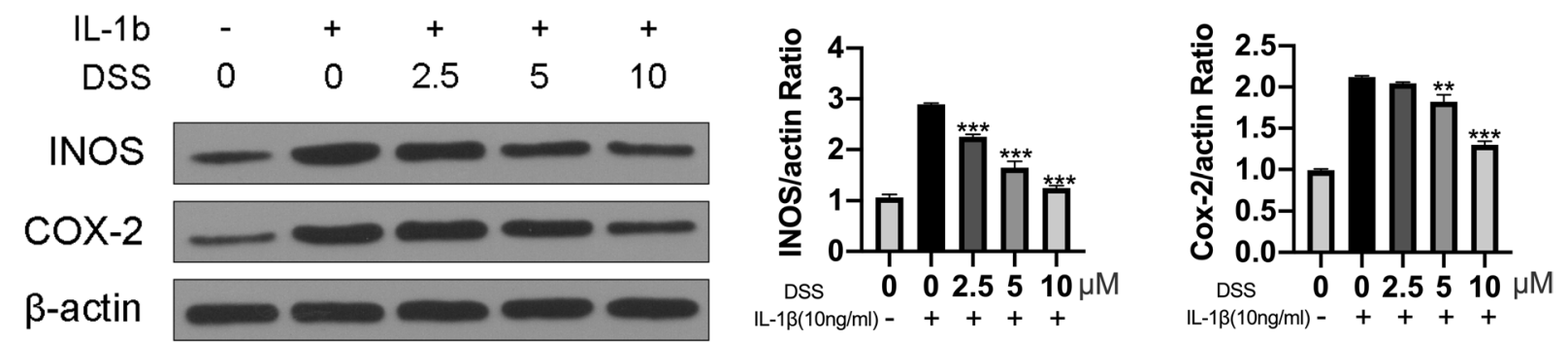

(C)
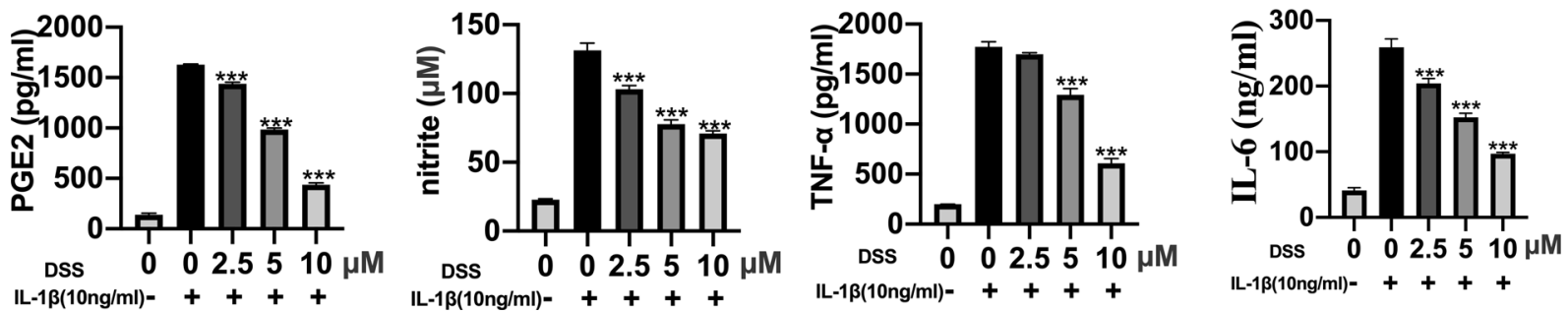

Fig. 2 DSS inhibited IL-1 $\beta$-induced chondrocytes inflammation. The chondrocytes were pretreated with 2.5, 5, and $10 \mu \mathrm{M}$ DSS for $24 \mathrm{~h}$ and stimulated with $10 \mathrm{ng} / \mathrm{ml} \mathrm{IL}-1 \beta$ for $2 \mathrm{~h}$. A The mRNA levels of inflammation-related markers IL-6, COX-2, TNF-a, and iNOS were evaluated by qRT-PCR. B The protein levels of iNOS and COX-2 was detected by Western blot with $\beta$-actin as the internal reference. C The production of PGE2, TNF-a, NO, and IL-6 were detected by ELISA. ${ }^{* *} \mathrm{p}<0.01 ;{ }^{* *} \mathrm{p}<0.001$

disease (Han 2019) and Lewis lung carcinoma xenografts in mice (Cao 2019). In addition, DSS has anti-tumor activity in B16F10 melanoma by inhibiting angiogenesis and tumor cell invasion (Zhang 2010). Recently, DSS has been reported to have anti-immune activity (Ye 2020; Guan 2015), which attracted us to focus on its role in OA progression. Interestingly, we found that
DSS treatment significantly decreased IL- $1 \beta$-enhanced expression of IL-6, COX-2, TNF- $\alpha$, and iNOS in a dosedependent manner. Meanwhile, DSS decreased IL-1 $\beta$ induced expression of inflammatory makers iNOS and COX-2 at the protein level. Moreover, IL-1 $\beta$ upregulated MMP3, MMP13, ADAMTS4, and ADAMTS5 and downregulated collagen II and aggrecan, while these effects

(See figure on next page.)

Fig. 3 DSS protected chondrocytes against IL-1 $\beta$-induced ECM degradation. The chondrocytes were treated with 2.5, 5, and $10 \mu \mathrm{M}$ DSS for $24 \mathrm{~h}$ and then stimulated by $10 \mathrm{ng} / \mathrm{ml} \mathrm{IL-1 \beta}$ for $2 \mathrm{~h}$. A, B Protein levels of MMP3, MMP13, ADAMTS4, ADAMTS5, aggrecan, and collagen II in chondrocytes (A), and their quantification by the Quantity ONE software (B). C, D Representative fluorescence images of collagen II and MMP3 detected by immunofluorescent staining assay $(\mathbf{C})$ and the percentage of cell immunofluorescence in chondrocytes that were pretreated with $10 \mu \mathrm{M}$ DSS for $24 \mathrm{~h}$ and stimulated by $10 \mathrm{ng} / \mathrm{ml} \mathrm{IL-1 \beta}$ for $2 \mathrm{~h}$ (D). Scale bar $=50 \mu \mathrm{m}$. ${ }^{*} \mathrm{p}<0.05,{ }^{* *} \mathrm{p}<0.01$, and ${ }^{* * *} \mathrm{p}<0.001$ 
(A)

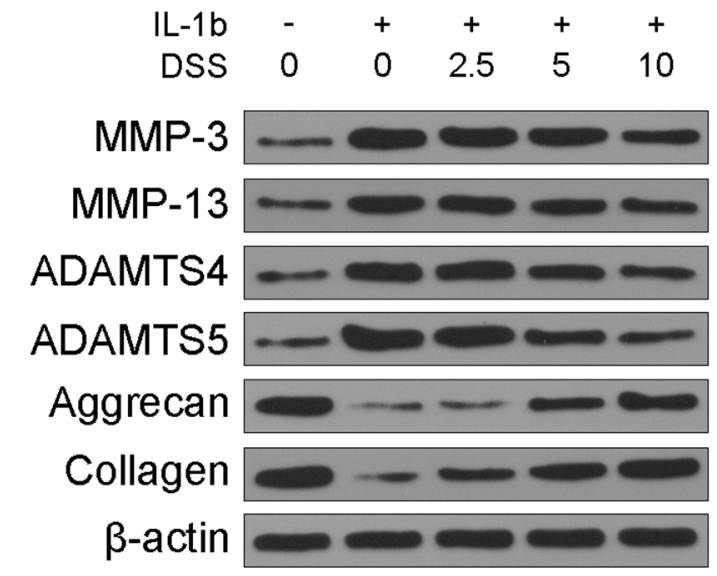

(C)

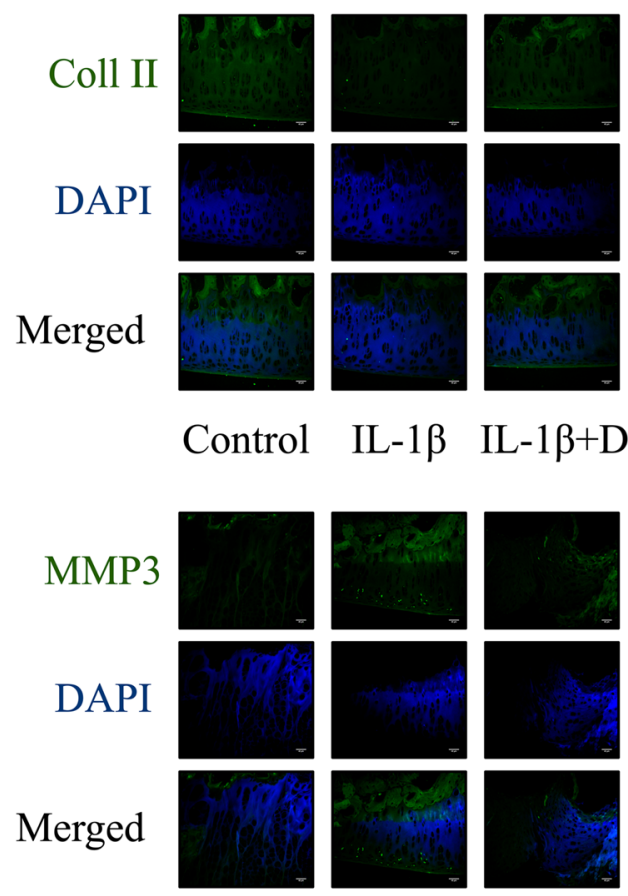

Control IL-1 $\beta$ IL-1 $\beta+$ DSS
(B)
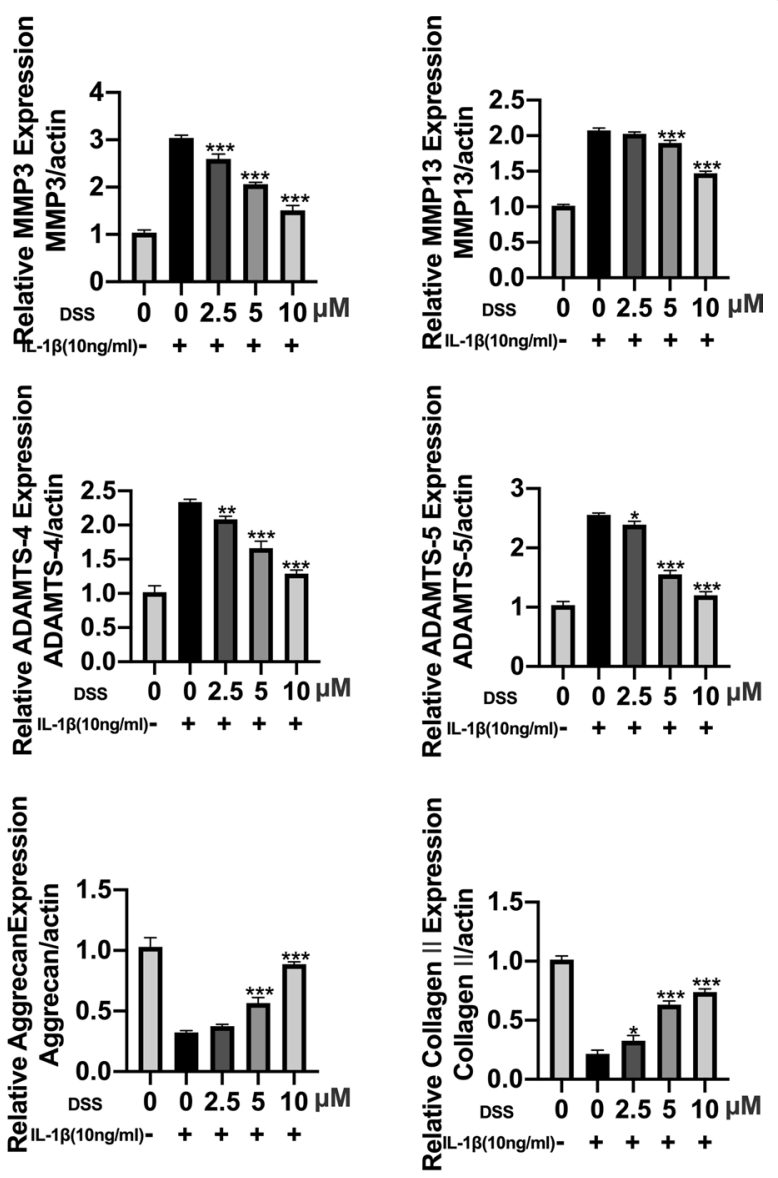

(D)
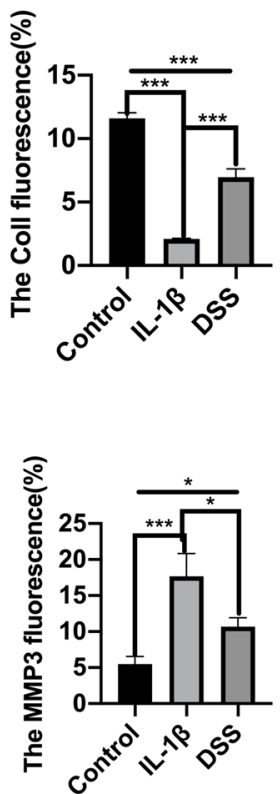

Fig. 3 (See legend on previous page.) 
(A)

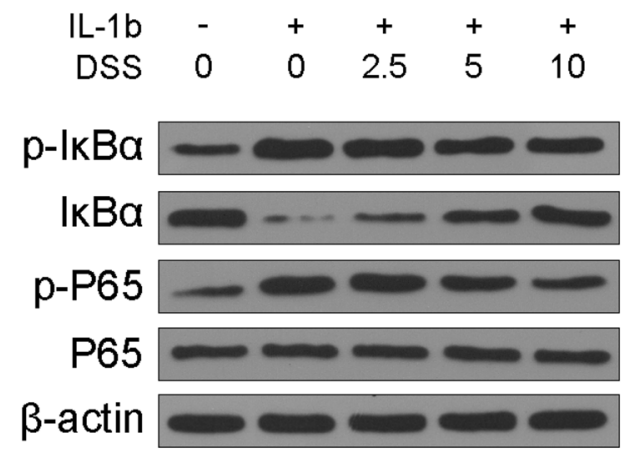

(C)

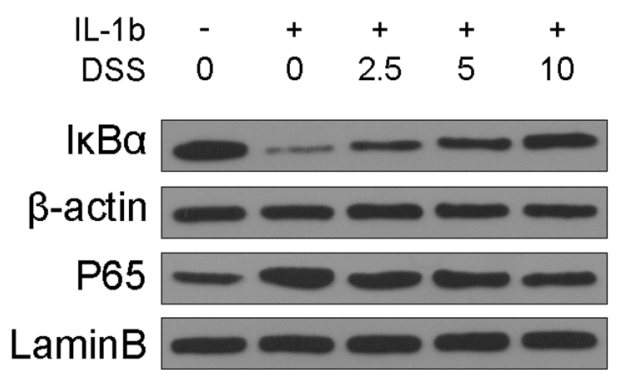

(B)
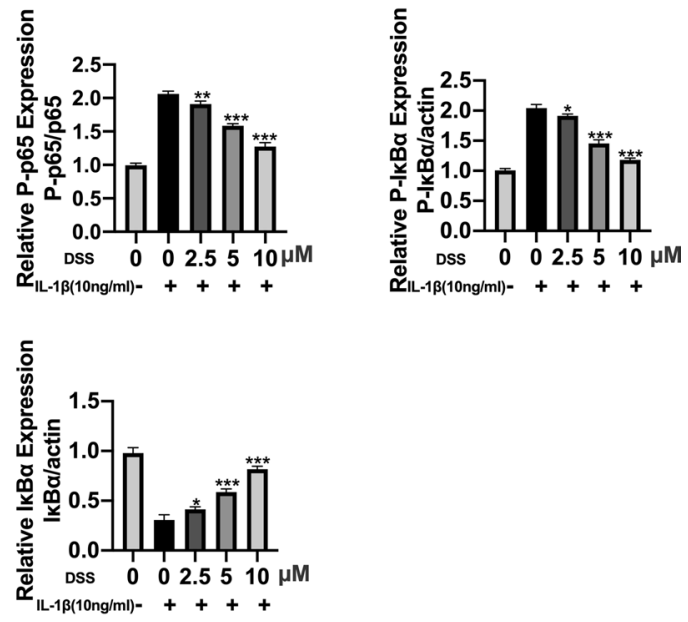

Cytoplasm

\section{Nucleus}

(D)
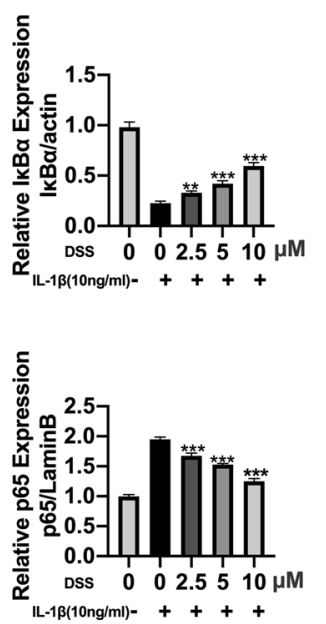

Fig. 4 DSS inhibited IL-1 $\beta$-induced activation of the NF-KB pathway in chondrocytes. The chondrocytes were treated 2.5, 5 and $10 \mu \mathrm{M}$ DSS for $24 \mathrm{~h}$ and then induced with or without $10 \mathrm{ng} / \mathrm{ml} \mathrm{IL}-1 \beta$ for $2 \mathrm{~h}$. The protein levels of p65, p-p65, IKBa, and p-IKBa were detected by Western blot (A) and quantitative analysis (B). The protein levels of $1 \mathrm{kBa}$ in the cytoplasm and p65 in the nucleus of chondrocytes were detected by Western blot (C) and quantitative analysis (D). ${ }^{*} p<0.05,{ }^{* *} p<0.01$, and ${ }^{* *} p<0.001$

were significantly reversed by DSS treatment. All these data demonstrated that DSS efficiently inhibited IL-1 $\beta$ induced inflammatory responses and ECM degradation in chondrocytes, suggesting that DSS might be a potential therapeutic agent for OA.

Previous studies have identified several signaling pathways involving the action mechanism of IL-1 $\beta$ in OA progression, such as p38 MAPK signaling pathway (Wang 2021), PI3K/Akt/mTOR signaling pathway (Xu 2021), nuclear factor erythroid 2-related factor-2/ heme oxygenase-1 signaling pathway (Zhu 2021), JAK2/STAT3 signaling pathway (Liu et al. 2020), and $\mathrm{Wnt} / \beta$-catenin signaling pathway (Miyatake and Kumagai 2020). Among them, NF- $\kappa B$ signaling is a widely studied pathway participating in IL-1 $\beta$-induced inflammatory response during OA development (Jimi et al. 2019; Choi et al. 2019). Based on the effects of DSS on inflammatory responses, we focused on the NF- $\mathrm{KB}$ signaling pathway. Our results indicated that DSS significantly inhibited p65 phosphorylation and nuclear translocation and promoted IкB $\alpha$ level in the cytoplasm, suggesting a suppressive effect of DSS on activation of NF-kB signaling pathway in chondrocytes. However, whether one or more signaling pathways mediate the effects of DSS during OA progression should be further determined.

DMM model is a widely used in vivo model for studies on the pathogenic processes during OA progression 


\section{(A)}
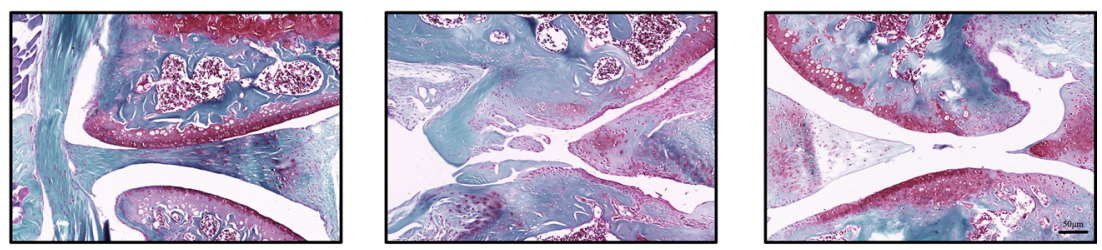

SafraninO
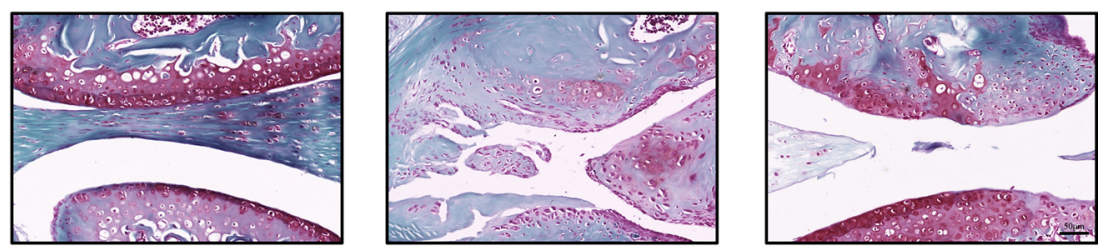

$\mathrm{H} \& \mathrm{E}$
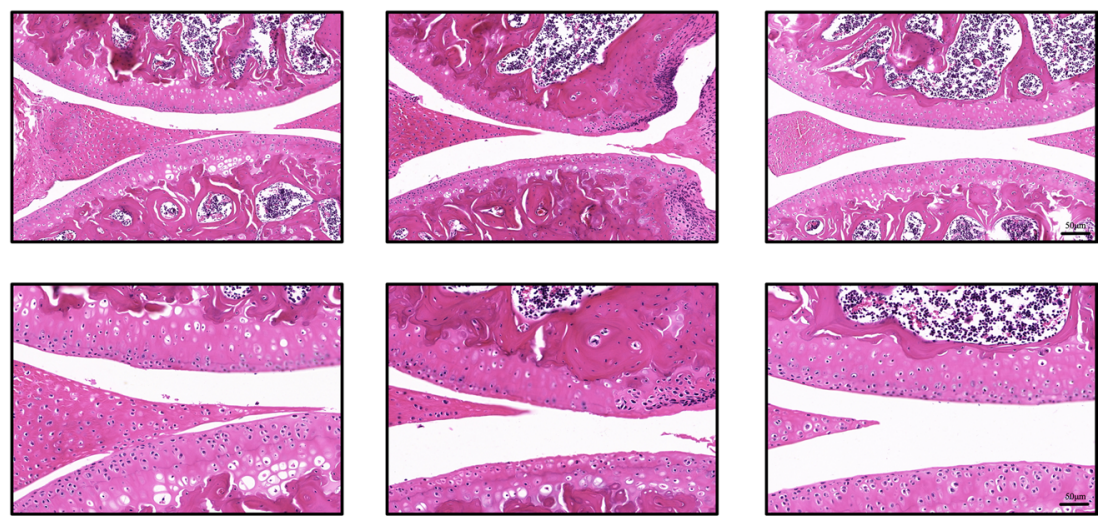

DMM

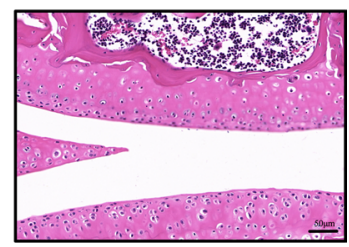

Sham

$\mathrm{DMM}+\mathrm{DSS}$

(B)

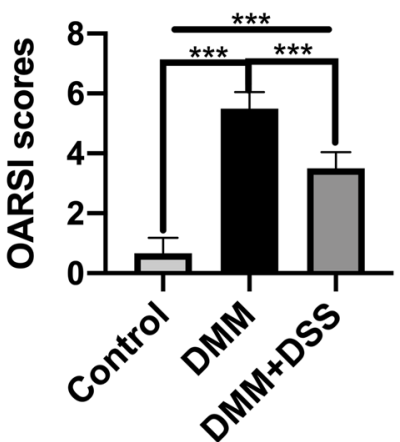

Fig. 5 DSS attenuated OA progression in mice model. A Representative images of safranin O and H\&E staining of cartilage sections from different groups. B The cartilage OARSI scores of different groups. Scale bar $=50 \mu \mathrm{m}$. Ten mice in each group, ${ }^{* * *} \mathrm{p}<0.001$

(Glasson 2007). To determine the potential protective role of DSS in OA, an in vivo mouse model was constructed. As expected, DSS efficiently attenuated OA progression in the mouse model. To better define the role of NF- $\kappa B$ signaling in the functions of DSS in OA, in the future, we will apply specific NF- $\mathrm{kB}$ signaling inhibitors to further understand the specific mechanism of DSS in OA progression.

\section{Conclusion}

In summary, our results demonstrated that DSS reduced inflammatory responses in OA and efficiently attenuated OA progression in a mouse model, possibly via deactivating the NF- $\mathrm{kB}$ signaling pathway, which provided a potential treatment strategy for OA. 


\section{Abbreviations}

DSS: Danshensu; OA: Osteoarthritis; DMM: Destabilization of the medial menis-

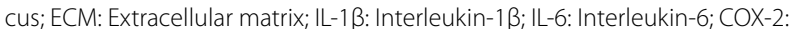
Cyclooxygenase 2; TNF-a: Tumor necrosis factor- $\alpha$; iNOS: Inducible nitric oxide synthase; MMP3: Matrix metalloproteinase 3; MMP13: Matrix metalloproteinase 13; ADAMTS4: A disintegrin and metalloproteinase with thrombospondin motifs 4; ADAMTS5: A disintegrin and metalloproteinase with thrombospondin motifs 5; NF-kB: Nuclear factor-kappa B.

\section{Acknowledgements}

Not applicable.

\section{Authors' contributions}

FC and WH designed the study. ZX carried out experiments and wrote the manuscript, $F C$ and $W H$ revised the paper, ZX, TK, YZ and LG collected patient specimens and related information. $Z X, T K, Y Z$ and $L G$ contributed to analysing the data. All authors reviewed the results and approved the final version of the manuscript.

\section{Funding}

Fujian Natural Science Foundation (2020J011099). Fujian Natural Science Foundation General Project (Project Number: 2020J011099).

\section{Availability of data and materials}

The analyzed data sets generated during the present study are available from the corresponding author on reasonable request.

\section{Declarations}

\section{Ethical approval and consent to participate}

The animal experiment was performed according to the National Institutes of Health Guide for Care and Use of Laboratory Animals and approved by Fujian Provincial Hospital.

\section{Consent for publication}

Not applicable.

\section{Competing interests}

The authors declared no conflict of interest.

\section{Author details}

${ }^{1}$ Shengli Clinical Medical College of Fujian Medical University, Fuzhou 350004, Fujian Province, People's Republic of China. ${ }^{2}$ Department of Emergency Surgery, Fujian Provincial Hospital, No.134 East Street, Gulou District, Fuzhou 350001, Fujian Province, People's Republic of China. ${ }^{3}$ Fujian Provincial Key Laboratory of Emergency Medicine, Fujian Provincial Hospital, Fuzhou 350001, Fujian, People's Republic of China.

Received: 6 December 2020 Accepted: 15 June 2021

Published online: 20 July 2021

\section{References}

Argoff CE. Recent developments in the treatment of osteoarthritis with NSAIDs. Curr Med Res Opin. 2011;27:1315-27. https://doi.org/10.1185/ 03007995.2011 .568058$.

Berenbaum F. Signaling transduction: target in osteoarthritis. Curr Opin Rheumatol. 2004;16:616-22. https://doi.org/10.1097/01.bor.00001 33663.37352.4a.

Brosseau $L$, et al. Thermotherapy for treatment of osteoarthritis. Cochrane Database Syst Rev. 2003. https://doi.org/10.1002/14651858.cd004522.

Cao G, et al. Danshensu, a novel indoleamine 2,3-dioxygenase1 inhibitor, exerts anti-hepatic fibrosis effects via inhibition of JAK2-STAT3 signaling. Phytomed: Int J Phytother Phytopharmacol. 2019;63: 153055. https://doi. org/10.1016/j.phymed.2019.153055.

Choi MC, Jo J, Park J, Kang HK, Park Y. NF-kB signaling pathways in osteoarthritic cartilage destruction. Cells. 2019. https://doi.org/10.3390/cells 8070734.
Conaghan PG, Cook AD, Hamilton JA, Tak PP. Therapeutic options for targeting inflammatory osteoarthritis pain. Nat Rev Rheumatol. 2019;15:355-63. https://doi.org/10.1038/s41584-019-0221-y.

Felson DT, Clinical practice. Osteoarthritis of the knee. N Engl J Med. 2006;354:841-8. https://doi.org/10.1056/NEJMcp051726.

Glasson SS. In vivo osteoarthritis target validation utilizing genetically-modified mice. Curr Drug Targets. 2007;8:367-76. https://doi.org/10.2174/ 138945007779940061.

Goldring SR, Goldring MB. Changes in the osteochondral unit during osteoarthritis: structure, function and cartilage-bone crosstalk. Nat Rev Rheumatol. 2016;12:632-44. https://doi.org/10.1038/nrrheum.2016.148.

Guan Y, et al. Effects and mechanism of combination of Rhein and Danshensu in the treatment of chronic kidney disease. Am J Chin Med. 2015;43:1381-400. https://doi.org/10.1142/s0192415×15500780.

Han B, et al. Neuroprotective effects of Danshensu in Parkinson's disease mouse model induced by 1-methyl-4-phenyl-1,2,3,6-tetrahydropyridine. Behav Pharmacol. 2019;30:36-44. https://doi.org/10.1097/fbp.00000 00000000412.

Jia Y, et al. Morusin ameliorates IL-1 beta-induced chondrocyte inflammation and osteoarthritis via NF-kappaB signal pathway. Drug Des Dev Ther. 2020;14:1227-40. https://doi.org/10.2147/dddt.s244462.

Jimi E, Fei H, Nakatomi C. NF-kB signaling regulates physiological and pathological chondrogenesis. Int J Mol Sci. 2019. https://doi.org/10.3390/ijms2 0246275 .

Jotanovic Z, Mihelic R, Sestan B, Dembic Z. Role of interleukin-1 inhibitors in osteoarthritis: an evidence-based review. Drugs Aging. 2012;29:343-58. https://doi.org/10.2165/11599350-000000000-00000.

Krustev E, Rioux D, McDougall JJ. Mechanisms and mediators that drive arthritis pain. Curr Osteoporos Rep. 2015;13:216-24. https://doi.org/10.1007/ s11914-015-0275-y.

Liu B, et al. Etoricoxib decreases subchondral bone mass and attenuates biomechanical properties at the early stage of osteoarthritis in a mouse model. Biomed Pharmacother. 2020a;127: 110144. https://doi.org/10. 1016/j.biopha.2020.110144.

Liu W, Chen Y, Zeng G, Yang T, Song W. INSR mediated by transcription factor KLF4 and DNA methylation ameliorates osteoarthritis progression via inactivation of JAK2/STAT3 signaling pathway. Am J Trans Res. 2020b;12:7953-67.

McDougall JJ, Andruski B, Schuelert N, Hallgrimsson B, Matyas JR. Unravelling the relationship between age, nociception and joint destruction in naturally occurring osteoarthritis of Dunkin Hartley guinea pigs. Pain. 2009;141:222-32. https://doi.org/10.1016/j.pain.2008.10.013.

Mendes AF, Carvalho AP, Caramona MM, Lopes MC. Role of nitric oxide in the activation of NF-kappaB, AP-1 and NOS II expression in articular chondrocytes. Inflamm Res. 2002;51:369-75. https://doi.org/10.1007/pl00000317.

Mitchell JP, Carmody RJ. NF-kappaB and the transcriptional control of inflammation. Int Rev Cell Mol Biol. 2018;335:41-84. https://doi.org/10.1016/bs. ircmb.2017.07.007.

Miyatake K, Kumagai K. Sclerostin inhibits interleukin-1 $\beta$-induced late stage chondrogenic differentiation through downregulation of Wnt/ $\beta$-catenin signaling pathway. PLoS ONE. 2020;15: e0239651. https://doi.org/10. 1371/journal.pone.0239651.

Nelson AE. Osteoarthritis year in review 2017: clinical. Osteoarthritis Cartilage. 2018;26:319-25. https://doi.org/10.1016/j.joca.2017.11.014.

Pattoli MA, MacMaster JF, Gregor KR, Burke JR. Collagen and aggrecan degradation is blocked in interleukin-1-treated cartilage explants by an inhibitor of IkappaB kinase through suppression of metalloproteinase expression. J Pharmacol Exp Ther. 2005;315:382-8. https://doi.org/10. 1124/jpet.105.087569.

Pritzker KP, et al. Osteoarthritis cartilage histopathology: grading and staging. Osteoarthritis Cartilage. 2006;14:13-29. https://doi.org/10.1016/j.joca. 2005.07.014.

Ran J, et al. Schisandrin B ameliorated chondrocytes inflammation and osteoarthritis via suppression of NF-kappaB and MAPK signal pathways. Drug Des Dev Ther. 2018;12:1195-204. https://doi.org/10.2147/dddt.s162014.

$\operatorname{Rim}$ YA, Nam Y, Ju JH. The role of chondrocyte hypertrophy and senescence in osteoarthritis initiation and progression. Int J Mol Sci. 2020. https://doi. org/10.3390/ijms21072358.

Saklatvala J. Inflammatory signaling in cartilage: MAPK and NF-kappaB pathways in chondrocytes and the use of inhibitors for research 
into pathogenesis and therapy of osteoarthritis. Curr Drug Targets. 2007:8:305-13. https://doi.org/10.2174/138945007779940115.

Schmittgen TD, Livak KJ. Analyzing real-time PCR data by the comparative C(T) method. Nat Protoc. 2008;3:1 101-8. https://doi.org/10.1038/nprot. 2008.73.

Schomberg D, Ahmed M, Miranpuri G, Olson J, Resnick DK. Neuropathic pain: role of inflammation, immune response, and ion channel activity in central injury mechanisms. Ann Neurosci. 2012;19:125-32. https://doi.org/10. 5214/ans.0972.7531.190309.

Stanton $\mathrm{H}$, et al. ADAMTS5 is the major aggrecanase in mouse cartilage in vivo and in vitro. Nature. 2005;434:648-52. https://doi.org/10.1038/natur e03417.

Wang XP, et al. Quercetin suppresses apoptosis of chondrocytes induced by IL-1 $\beta$ via inactivation of $\mathrm{p} 38$ MAPK signaling pathway. Exp Ther Med. 2021;21:468. https://doi.org/10.3892/etm.2021.9899.

Wen XD, et al. Salvia miltiorrhiza (dan shen) significantly ameliorates colon inflammation in dextran sulfate sodium induced colitis. Am J Chin Med. 2013:41:1097-108. https://doi.org/10.1142/s0192415×13500742.

Xia T, et al. Trans-Cinnamaldehyde inhibits IL-1 beta-stimulated inflammation in chondrocytes by suppressing NF-kappaB and p38-JNK pathways and exerts chondrocyte protective effects in a rat model of osteoarthritis. BioMed Res Int. 2019;2019: 4039472. https://doi.org/10.1155/2019/40394 72.

Xu C, et al. FSTL1 promotes nitric oxide-induced chondrocyte apoptosis via activating the SAPK/JNK/caspase3 signaling pathway. Gene. 2020;732: 144339. https://doi.org/10.1016/j.gene.2020.144339.
Xu K, et al. SIRT3 ameliorates osteoarthritis via regulating chondrocyte autophagy and apoptosis through the PI3K/Akt/mTOR pathway. Int J Biol Macromol. 2021;175:351-60. https://doi.org/10.1016/j.jibiomac.2021.02. 029.

Ye T, et al. Inhibition of nuclear factor kappa B as a mechanism of Danshensu during Toll-like receptor 2-triggered inflammation in macrophages. Int Immunopharmacol. 2020;83: 106419. https://doi.org/10.1016/j.intimp. 2020.106419.

Yin W, Lei Y. Leonurine inhibits IL-1 beta induced inflammation in murine chondrocytes and ameliorates murine osteoarthritis. Int Immunopharmacol. 2018;65:50-9. https://doi.org/10.1016/j.intimp.2018.08.035.

Zhang LJ, et al. Danshensu has anti-tumor activity in B16F10 melanoma by inhibiting angiogenesis and tumor cell invasion. Eur J Pharmacol. 2010;643:195-201. https://doi.org/10.1016/j.ejphar.2010.06.045.

Zhang R, et al. Protective effects of sweroside on IL-1 beta-induced inflammation in rat articular chondrocytes through suppression of NF-kappaB and mTORC1 signaling pathway. Inflammation. 2019;42:496-505. https://doi. org/10.1007/s10753-018-0906-4.

Zhu DC, et al. Maltol inhibits the progression of osteoarthritis via the nuclear factor-erythroid 2-related factor-2/heme oxygenase-1 signal pathway in vitro and in vivo. Food Funct. 2021;12:1327-37. https://doi.org/10. 1039/dofo02325f

\section{Publisher's Note}

Springer Nature remains neutral with regard to jurisdictional claims in published maps and institutional affiliations.
Ready to submit your research? Choose BMC and benefit from:

- fast, convenient online submission

- thorough peer review by experienced researchers in your field

- rapid publication on acceptance

- support for research data, including large and complex data types

- gold Open Access which fosters wider collaboration and increased citations

- maximum visibility for your research: over 100M website views per year

At BMC, research is always in progress.

Learn more biomedcentral.com/submissions 